\title{
Thoracoscopic removal of a displaced thoracoamniotic shunt in a newborn with antenatal pleural effusion-a case report
}

\author{
Goby T. W. Sham ${ }^{1}$, Patrick H. Y. Chung ${ }^{1}$, Iris M. C. Chan ${ }^{2}$, W. C. Leung ${ }^{3}$, Kenneth K. Y. Wong ${ }^{1}$ \\ ${ }^{1}$ Department of Surgery, Li Ka Shing Faculty of Medicine, The University of Hong Kong, Hong Kong, China; ${ }^{2}$ Department of Paediatrics, Kwong \\ Wah Hospital, Hong Kong, China; ${ }^{3}$ Department of Obstetrics and Gynaecology, Kwong Wah Hospital, Hong Kong, China \\ Correspondence to: Dr. Patrick H. Y. Chung. Department of Surgery, The University of Hong Kong, Queen Mary Hospital, 102 Pokfulam Road, \\ Hong Kong, China. Email: chungphy@hku.hk.
}

\begin{abstract}
Thoracoamniotic shunt has been considered as a treatment for antenatal pleural effusion and complication is rare. In majority of cases, the shunt can be removed uneventfully. In this article, we reported a rare complication of shunt migration resulting in the need of thoracoscopic removal at newborn period. The patient born at 39+3 weeks of gestation suffered from antenatal chylothorax detected at 28 weeks and was managed by intrauterine thoracoamniotic shunt insertion. This was complicated by shunt displacement, which caused respiratory distress after birth requiring ventilatory support and progressive pleural effusion in this patient. To prevent further neonatal compromise, thoracoscopic removal of the retained shunt was done on day 7 of life followed by post-op chest drain insertion. Post-op condition was stable with resolution of respiratory distress, and the patient was discharge on post-op day 16 . We would like to remind clinicians about this potential complication of thoracoamniotic shunt, which can pose a potential risk of severe neonatal compromise, and that it can be managed by minimal invasive surgery even in the newborn period.
\end{abstract}

Keywords: Thoracoamniotic shunt displacement; congenital chylothorax; thoracoscopy; case report

Submitted Feb 21, 2020. Accepted for publication Aug 16, 2020.

doi: $10.21037 / \mathrm{tp}-20-74$

View this article at: http://dx.doi.org/10.21037/tp-20-74

\section{Introduction}

Thoracoamniotic shunt is a well-recognised treatment for antenatal pleural effusion for its effectiveness and low complication rate (1). Although rare, shunt complication can cause severe foetal and neonatal compromise. A reported rate of foetal death after shunt insertion is $38 \%$ (2). In particular, shunt displacement can jeopardize fetal pulmonary development and cause severe neonatal compromise. Despite its clinical importance, only sporadic cases have been reported due to its rarity, let alone its management. Thoracoscopic surgery is a known approach for this complication. A few case reports documented its effectiveness and satisfactory outcome. The benefits of a minimally invasive surgery (MIS), even in the newborn period, also prove its value in the management of this complication. We hereby report a case of antenatal chylothorax managed by thoracoamniotic shunt insertion with subsequently shunt displacement. This complication was successfully managed by thoracoscopic surgery without residual problem. We present the following case in accordance with the CARE reporting checklist (available at http://dx.doi.org/10.21037/tp-20-74).

\section{Case presentation}

Our patient is a full-term $39+3$ weeks baby girl with antenatal history of left pleural effusion first detected at 26 weeks. At $29+1$ weeks, the effusion measured $2.8 \mathrm{~cm}$ depth on ultrasound scan with evidence of mediastinal shift. At $29+3$ weeks, amniocentesis together with ultrasoundguided intrauterine thoracocentesis was performed and $36 \mathrm{~mL}$ of straw-colored fluid was aspirated. Amniotic and pleural fluid analysis were unremarkable. However, pleural effusion re-accumulated within two days post-tapping and intrauterine shunting was decided to prevent recurrence 


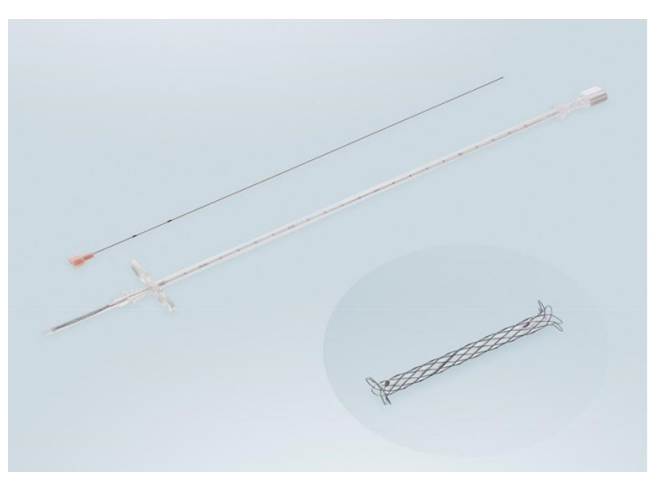

Figure 1 Somatex ${ }^{\circledR}$ intrauterine shunt with introducers for intrauterine insertion.

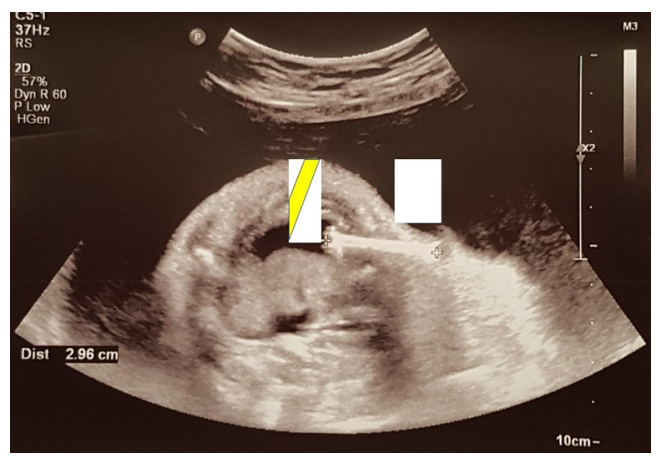

Figure 2 Fetal ultrasound scan showing the position of the shunt. The white box shows the thoracoamniotic shunt being inserted in a suboptimal direction (oblique rather than perpendicular to foetal chest wall); the yellow arrow shows the optimal direction of insertion.

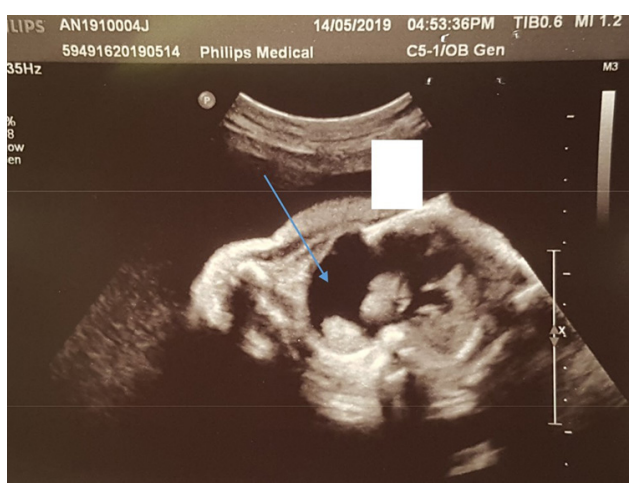

Figure 3 Migration of shunt into foetal chest wall causing blockage of shunt (white) with re-accumulation of pleural effusion (blue) on day 10 after shunt insertion, 31 weeks of gestation. before birth. At 29+5 weeks, an intrauterine SOMATEX ${ }^{\circledR}$ thoracoamniotic shunt was inserted under ultrasound guidance (Figure 1). The procedure was complicated by fetal movements and the positioning of the shunt was hence suboptimal with an oblique rather than the usual perpendicular direction across the fetal chest wall (Figure 2). Despite such circumstance, the shunt was still functioning partially with follow-up USG at 30 weeks showing decrease in left pleural effusion to $1 \mathrm{~cm}$ depth and reduced mediastinal shift. However, internal shunt migration was noted at 31 weeks with re-accumulation of pleural fluid (Figure 3). At $33+1$ weeks, progressive reaccumulation of $3.2 \mathrm{~cm}$ pleural effusion was detected. Nonetheless, parents opted for conservative treatment. Despite shunt displacement, pleural effusion resolved spontaneously as the fetus approaching term and at $38+3$ weeks, only tiny residual effusion was noted. The baby was delivered in her parent hospital by normal spontaneous vaginal delivery uneventfully at 39+3 weeks. Apgar score 9 at $1 \mathrm{~min}$ and 10 at $5 \mathrm{~min}$. Mild respiratory distress was noted on newborn examination. Chest examination showed minimal subcostal in-sucking and there was skin budging with tip of the shunt visible under the right anterior chest wall. Chest $\mathrm{X}$-ray after delivery showed that the shunt was extending from right parasternal 3 rd intercostal space to left pleural cavity and there was no significant pleural effusion. The baby was subsequently put on nasal continuous positive airway pressure (CPAP). Respiratory distress resolved on day 1 of life and CPAP was weaned off to room air. Feeding with normal formula was started on the same day. However, pleural effusion re-accumulated to $7 \mathrm{~mm}$ and failed to resolve with conservative treatment. The patient was transferred to pediatric surgical unit for further management on day 4 of life.

After evaluation of the patient's condition and shunt position, thoracoscopic removal of the retained thoracoamniotic shunt was scheduled on day 5 of life. Under $5 \mathrm{~mm}$ thoracoscopy, the position of the shunt was confirmed at the subcutaneous layer anterior to sternum. The end of the shunt was seen inside the thoracic cavity with no impingement or injury to the underlying lung parenchyma or major vessels (Figure 4). The entire shunt was exposed and mobilized from surrounding tissue. The retrieval of the shunt was done under direct vision with a $2 \mathrm{~cm}$ skin incision made. The procedure lasted for $49 \mathrm{~min}$ and no intra-operative complication encountered. A Fr 16 left chest drain was placed post-operatively to drain the residual pleural effusion which was subsequently confirmed 


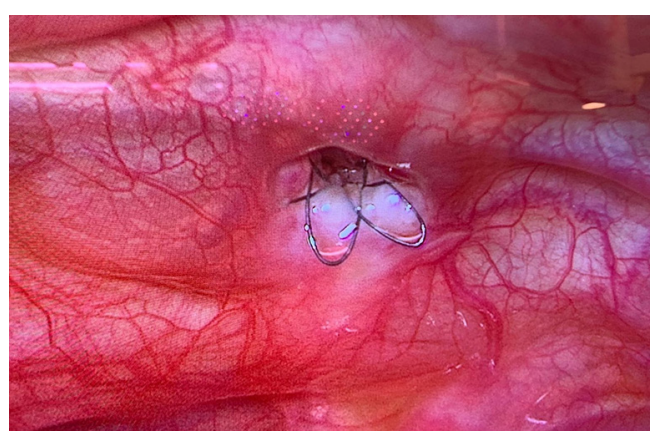

Figure 4 Thoracoscopic view of the displaced shunt which was located inside the thoracic cavity with part of it embedded in the chest wall without any impingement or injury to the underlying lung parenchyma or major vessels.

as chylous.

Post operatively, the patient was stable and was transferred back to her parent hospital with a left chest chain for further management of chylothorax. Medium chained triglyceride (MCT) rich formula was started and full enteral feeding was achieved on day 11. USG showed no re-accumulation of left pleural effusion and the chest drain was removed on day 13 of life. She remained stable and was discharged on post-op day 16. A timeline is provided to summarise the case progression (Figure 5).

All procedures performed in studies involving human participants were in accordance with the ethical standards of the institutional research committee and with the Helsinki Declaration (as revised in 2013). Written informed consent was obtained from the parents of the patient.

\section{Discussion}

Antenatal pleural effusion can cause undesirable consequences to the neonates, affecting normal lung development and perinatal survival. While some may resolve spontaneously, intervention is indicated when the pleural effusion is persistently large or when there is the presence of hydrops fetalis, indicating impending fetal compromise $(1,3)$. Our patient had progressive pleural effusion over 2 weeks with mediastinal shift, justifying surgical intervention in this case. Placement of thoracoamniotic shunt has been shown to improve Apgar scores, duration of mechanical ventilations and perinatal complications (4). Despite its benefits, complications and risks exist. Complications include dislodgement of catheter, thrombus occlusion of catheter, fatal fetal hemorrhage, procedure-related abruptio placentae, premature rupture of membranes and preterm labor (5). A possible mechanism of shunt displacement was hypothesized to be internalization of the shunt due to growing chest size of the fetus or due to fetal respiratory movement (1). In our patient, an additional risk of shunt displacement can be due to suboptimal intrauterine shunt insertion due to fetal movements. In one case series conducted in Chile with patients selected from three centers, 3 out of 13 fetuses who had a thoracoamniotic shunt inserted was complicated by dislodgement of the shunt, giving a prevalence of $23 \%$ for such complication (6). Diagnosis of shunt displacement during fetal period is made by ultrasound. Prompt intervention of a displaced thoracoamniotic shunt is justified by its potential risk of severe neonatal compromise. One case series reported a case of shunt displacement compressing the lung hilar causing neonatal demise (7). Another case reported in 2016 presented a patient with congenital pulmonary airway malformation and foetal hydrops treated with thoracoamniotic shunt. Subsequent internal displacement of the shunt eventually resulted in refractory tension pneuemothorax (8). Clinicians should be aware of the disastrous effect of this complication and the importance of early surgical intervention.

For an uncomplicated thoracoamniotic shunt, the shunt should be clamped and removed immediately after birth to prevent pneumothorax according to NICE guideline (9). Yet, the ideal management and timing of intervention for a displaced thoracoamniotic shunt is not well documented due to its rarity. Only sporadic cases of thoracoscopic removal of a retained shunt were reported $(10,11)$. In the case series reported by the Japan group, the procedures were done several days after birth for stabilization of the respiratory status of the newborns. A similar strategy was applied in our patient in which the operation was done on day 5 of life for initial stabilization. However, we did not delay the operation a longer period to minimize the risk of fatal hilar compression and inflammatory response induced by the dislodged shunt.

MIS is known to provide more benefits and less morbidity and mortality than open surgery, namely less wound pain, fewer major wound complications, faster recovery, improved cosmesis and similar outcome achieved by open surgery (12). With recent advancement in instrumentation and experience, more and more pediatric surgeries are slowly adopting a minimally invasive technique from open surgery and these could be done even in newborn babies. For example, for pediatric thoracoscopic 


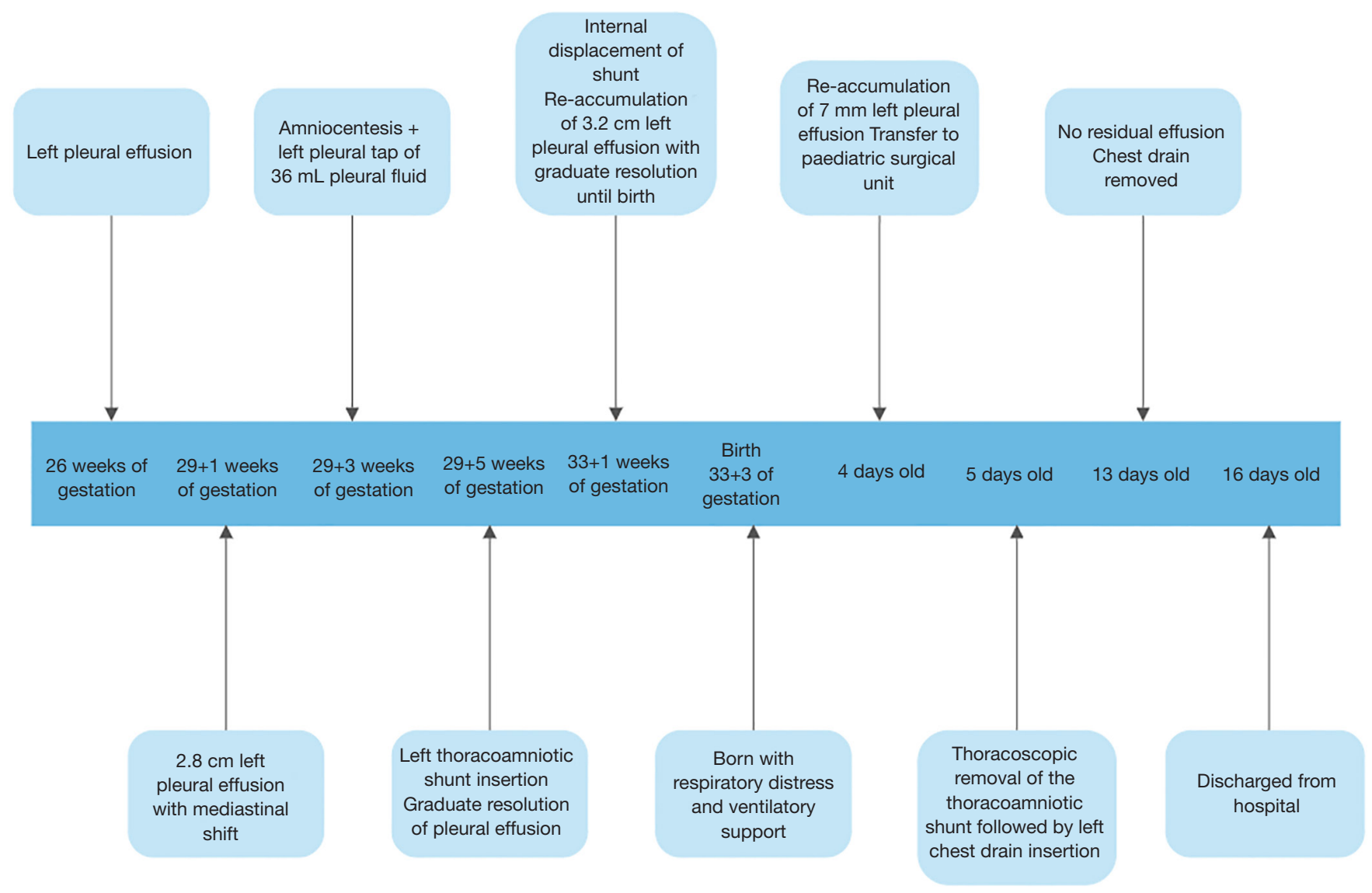

Figure 5 Progression of the case according to time line.

surgery, congenital diaphragmatic hernia $(\mathrm{CDH})$ has been increasingly managed by thoracoscopic assisted repair than the traditional open repair due to its intra-op and postop benefits $(13,14)$. The growing use of MIS in paediatric surgery has been extending across different subspecialties, from cardiac, urological, gastrointestinal surgeries to even thoracic and abdominal oncological surgeries in recent years (15). MIS is no doubt a promising surgical approach in pediatric surgery. The beauty of MIS procedure has been demonstrated in our patients who enjoyed a rapid recovery from the surgery with a small scar only.

In conclusion, antenatal pleural effusion can cause disastrous consequences to the neonates' pulmonary development and can be treated promptly by a thoracoamniotic shunt. However, shunt displacement could be a potential complication and should be monitored. With advances in MIS, displaced shunt can be removed even in newborn period by thoracoscopic surgery.

\section{Acknowledgments}

Funding: None.

\section{Footnote}

Reporting Checklist: The authors have completed the CARE reporting checklist. Available at http://dx.doi.org/10.21037/ tp-20-174

Conflicts of Interest: All authors have completed the ICMJE uniform disclosure form (available at http://dx.doi. org/10.21037/tp-20-74). PHYC serves as an unpaid editorial board member of Translational Paediatrics from Aug 2020 to Jul 2022. The other authors have no conflicts of interest to declare.

Ethical Statement: The authors are accountable for all aspects of the work in ensuring that questions related 
to the accuracy or integrity of any part of the work are appropriately investigated and resolved. Ethics approval is waived for case report study and this study. All procedures performed in studies involving human participants were in accordance with the ethical standards of the institutional research committee and with the Helsinki Declaration (as revised in 2013). Written informed consent was obtained from the parents of this patient for publication of this manuscript and any accompanying images. A copy of the written consent is available for review by the Editor-inChief of this manuscript.

Open Access Statement: This is an Open Access article distributed in accordance with the Creative Commons Attribution-NonCommercial-NoDerivs 4.0 International License (CC BY-NC-ND 4.0), which permits the noncommercial replication and distribution of the article with the strict proviso that no changes or edits are made and the original work is properly cited (including links to both the formal publication through the relevant DOI and the license). See: https://creativecommons.org/licenses/by-nc-nd/4.0/.

\section{References}

1. Alkazaleh F, Saleem M, Badran E. Intrathoracic displacement of pleuroamniotic shunt after successful in utero treatment of fetal hydrops secondary to hydrothorax. Case report and review of the literature. Fetal Diagn Ther 2009;25:40-3.

2. Wilson RD, Johnson MP. Prenatal ultrasound guided percutaneous shunts for obstructive uropathy and thoracic disease. Semin Pediatr Surg 2003;12:182-9.

3. Daltro P, Werner H, Gasparetto TD, et al. Congenital chest malformations: a multimodality approach with emphasis on fetal MR imaging. Radiographics 2010;30:385-95.

4. Carr BD, Sampang L, Church JT, et al. Fetal intervention for congenital chylothorax is associated with improved outcomes in early life. J Surg Res 2018;231:361-5.

\footnotetext{
Cite this article as: Sham GTW, Chung PHY, Chan IMC, Leung WC, Wong KKY. Thoracoscopic removal of a displaced thoracoamniotic shunt in a newborn with antenatal pleural effusion-a case report. Transl Pediatr 2020;9(5):702-706. doi: $10.21037 /$ tp-20-74
}

5. Mann S, Wilson RD, Bebbington MW, et al. Antenatal diagnosis and management of congenital cystic adenomatoid malformation. Semin Fetal Neonatal Med 2007;12:477-81.

6. Sepulveda W, Galindo A, Sosa A, et al. Intrathoracic dislodgement of pleuro-amniotic shunt. Three case reports with long -term follow-up. Fetal Diagn Ther 2005;20:102-5.

7. Blanch G, Walkinshaw SA, Hawdon JM, et al. Internalization of pleuroamniotic shunt causing neonatal demise. Fetal Diagn Ther 1996;11:32-6.

8. Law BH, Bratu I, Jain V, et al. Refractory tension pneumothorax as a result of an internally displaced thoracoamniotic shunt in an infant with a congenital pulmonary airway malformation. BMJ Case Rep 2016;2016:bcr2016216324.

9. The procedure: Insertion of pleuro-amniotic shunt for fetal pleural effusion: Guidance. Available online: https:// www.nice.org.uk/guidance/ipg190/chapter/2-Theprocedure

10. Macchini F, Gentilino V, Morandi A, et al. Thoracoscopic removal of retained thoracoamniotic shunt catheters in newborns. J Laparoendosc Adv Surg Tech A 2014;24:827-9.

11. Inoue S, Odaka A, Baba K, et al. Thoracoscopy-assisted removal of a thoracoamniotic shunt double-basket catheter dislodged into the fetal thoracic cavity: report of three cases. Surg Today 2014;44:761-6.

12. Ure BM, Jesch NK, Glüer S. What's new in minimally invasive paediatric surgery? Eur J Pediatr Surg 2002;12:361-5.

13. Chan E, Wayne C, Nasr A. Minimally invasive versus open repair of Bochdalek hernia: a meta-analysis. J Pediatr Surg 2014;49:694-9.

14. Qin J, Ren Y, Ma D. A comparative study of thoracoscopic and open surgery of congenital diaphragmatic hernia in neonates. J Cardiothorac Surg 2019;14:118.

15. Peycelon M, Audry G, Irtan S. Minimally invasive surgery in childhood cancer: a challenging future. Eur J Pediatr Surg 2014;24:443-9. 